\title{
Where are all the gravastars? Limits upon the gravastar model from accreting black holes
}

\section{Citation}

Broderick, Avery E, and Ramesh Narayan. 2007. "Where Are All the Gravastars? Limits upon the Gravastar Model from Accreting Black Holes." Classical and Quantum Gravity 24 (3): 659-66. https://doi.org/10.1088/0264-9381/24/3/009.

\section{Permanent link}

http://nrs.harvard.edu/urn-3:HUL.InstRepos:41384931

\section{Terms of Use}

This article was downloaded from Harvard University's DASH repository, and is made available under the terms and conditions applicable to Other Posted Material, as set forth at http:// nrs.harvard.edu/urn-3:HUL.InstRepos:dash.current.terms-of-use\#LAA

\section{Share Your Story}

The Harvard community has made this article openly available.

Please share how this access benefits you. Submit a story.

Accessibility 


\title{
Where are all the gravastars? Limits upon the gravastar model from accreting black holes
}

\author{
Avery E. Broderick ${ }^{1} \&$ Ramesh Narayan ${ }^{1}$ \\ ${ }^{1}$ Institute for Theory and Computation, Harvard-Smithsonian Center for \\ Astrophysics, MS 51, 60 Garden Street, Cambridge, MA 02138, USA \\ E-mail: abroderick@cfa.harvard.edu, rnarayan@cfa.harvard.edu
}

\begin{abstract}
The gravastar model, which postulates a strongly correlated thin shell of anisotropic matter surrounding a region of anti-de Sitter space, has been proposed as an alternative to black holes. We discuss constraints that present-day observations of well-known black hole candidates place on this model. We focus upon two black hole candidates known to have extraordinarily low luminosities: the supermassive black hole in the Galactic Center, Sagittarius A*, and the stellar-mass black hole, XTE J1118+480. We find that the length scale for modifications of the type discussed in Chapline et al. (2003) must be sub-Planckian.
\end{abstract}

PACS numbers: $\quad$ 04.70.-s, 04.80.Cc, 95.36.+x, 95.85.Hp, 95.85.Mt, 95.85.Nv, 98.35.Jk 


\section{Introduction}

There is strong observational evidence that many massive stars end their lives as dark, compact objects with masses larger than $3 M_{\odot}$ (Narayan 2005). In the context of general relativity, the only possible stable configuration for such an object is a black hole. Recently, Broderick \& Narayan (2006) demonstrated that the current near-infrared flux limits on the black hole candidate Sagittarius A* (Sgr A*) at the Galactic Center imply the existence of a horizon in this object. Their argument assumed only that any non-horizon model has reached steady state under continued accretion and that general relativity is an acceptable description of gravity outside the alternative object's photosphere.

In recent years a class of alternatives to general relativity has been advanced in which a large-scale quantum phase transition occurs during stellar collapse, preventing the formation of a horizon (Mazur \& Mottola 2001, Chapline et al. 2003, Visser \& Wiltshire 2004, Carter 2005, Lobo 2006). In the most developed of these "gravastar" models, general relativity is an emergent theory failing at small length scales.

Necessarily, these models are characterized by a large surface red-shift, enabling them to mimic black holes.

In principle, the gravastar model can escape the argument presented in Broderick \& Narayan (2006) by possessing a large thermal capacity (Chapline et al. 2003) and therefore not reaching steady state. However, it is expected that most compact objects in astrophysics grow substantially in mass via accretion. Therefore, if some of these are gravastars, there is a natural mechanism for providing large amounts of energy to heat them up. Indeed, stellar mass black holes are expected to accrete substantial fractions of their mass during formation in the form of a fallback disk (see, e.g., Woosley et al. 2002, Fryer et al. 2002). Similarly, within the context of the standard heirarchical models of galaxy formation, supermassive black holes are expected to grow primarily by accretion (see, e.g., Soltan 1982, Yu \& Tremaine 2002, Di Matteo et al. 2005).

Here we discuss the constraints that may be placed upon the gravastar models by observations of black hole candidates. For the purpose of concreteness we restrict ourselves to the particular model presented by Chapline et al. (2003), but the arguments may be applied to dark energy stars generally. In sections 2 and 3 we discuss the thermal evolution of gravastars and present the observational constraints placed upon the parameters of the Chapline et al. (2003) gravastar model, respectively. Concluding remarks are contained in 4 .

\section{Thermal Evolution}

\subsection{Accretion Heating}

The heating of neutron stars via accretion has been well documented. In contrast, there is little evidence for similar heating in the case of black-hole candidates. Indeed, this fact has been used to argue for the presence of event horizons in these objects (McClintock 
et al. 2004, Broderick \& Narayan 2006). However, the absence of detectable heating may also be consistent with a gravastar if the heat capacity is large enough that it requires prodigious amounts of heat to produce small changes in temperature. Nevertheless, some level of accretion heating is unavoidable and, as we show, provides surprisingly strong constraints on the gravastar model.

In the model described in Chapline et al. (2003), general relativity is an emergent theory of an underlying Lorentz violating microphysical theory, failing at small lengthscales. In this context, the gravastar is the result of a BEC-like phase transition induced by strong gravity. The internal energy $U$ of such a gravastar is related to its mass $m=M / M_{\odot}$ in solar units and temperature $T$ by

$$
U=\mathcal{U} \xi^{-1} m^{3} T^{3}
$$

where $\mathcal{U} \simeq 5.8 \times 10^{34} \mathrm{erg} K^{-3}$, and $\xi=l / l_{\mathrm{pl}}$ is the length scale in Planck units at which general relativity fails to adequately describe gravity. Note that while the particular form of $U$ is dependent upon the microphysics underlying a gravastar or dark-energy star, given a postulated form of $U$ the argument described below will necessarily constrain the given model.

The large surface redshifts employed in gravastar models have two immediate implications: (i) the internal energy generated per unit rest mass accreted is very nearly $c^{2}$, i.e., the usual accretion efficiency factor $\eta \approx 1$ (and will be neglected henceforth), and (ii) the radiation emitted from the surface of the object should be almost a perfect blackbody (Broderick \& Narayan 2006). The energy evolution of an accreting gravastar is determined by

$$
\frac{d U}{d t}=\dot{M} c^{2}-L
$$

where $\dot{M}$ is the mass accretion rate and $L$ is the total luminosity. Thus, from equation (1), we estimate that a gravastar which starts at zero temperature and rapidly accretes a mass $\Delta m M_{\odot}$ will be heated to a temperature as observed at infinity of

$$
T_{\mathrm{h}} \simeq\left(\frac{M_{\odot} c^{2}}{\mathcal{U} m^{2}}\right)^{1 / 3} \xi^{1 / 3}\left(\frac{\Delta m}{m}\right)^{1 / 3} \simeq 3.1 \times 10^{6} m^{-2 / 3} \xi^{1 / 3}\left(\frac{\Delta m}{m}\right)^{1 / 3} \mathrm{~K},
$$

where $m$ is the final mass. Here, "rapid accretion" means that the rate at which internal energy is added via accretion is much larger than the radiative luminosity $L$ of the heated surface, i.e., the mass accretion rate $\dot{M}$ satisfies

$$
\dot{M} \gg \frac{L}{c^{2}}=\frac{g \mathcal{A} \sigma}{c^{2}} m^{2} T_{\mathrm{h}}^{4} \simeq 1.1 \times 10^{-3} m^{-5 / 3} \xi^{4 / 3}\left(\frac{\Delta m}{m}\right)^{4 / 3} \dot{M}_{\mathrm{Edd}},
$$

where $\mathcal{A} m^{2}=108 \pi\left(G M_{\odot} / c^{2}\right)^{2}$ is the effective area of the radiating surface as measured at infinity, $g=29 / 8$ is a degeneracy factor ( 3 types of neutrinos + photons, assuming that the temperature in the local frame of the radiating surface is high enough for neutrinos to be emitted in thermal equilibrium), and $\dot{M}_{\text {Edd }}=2.3 \times 10^{-9} \mathrm{~m} M_{\odot} / \mathrm{yr}$ is the Eddington mass accretion rate, typically the maximum possible in astrophysical systems. 
In the opposite limit of slow accretion, the temperature is given by the equilibrium or steady state value:

$$
T_{\mathrm{eq}} \simeq\left[\frac{\dot{M} c^{2}}{g \mathcal{A} \sigma m^{2}}\right]^{1 / 4} \simeq 1.7 \times 10^{7}\left(\frac{\dot{M}}{\dot{M}_{\mathrm{Edd}}}\right)^{1 / 4} m^{-1 / 4} \mathrm{~K},
$$

where $\sigma$ is the Stefan-Boltzmann constant.

\subsection{Radiative Cooling}

Unless we find a gravastar that is currently accreting in steady state, it is generally insufficient to know the temperature to which accretion can heat it. Once accretion ceases, the surface will begin to cool via thermal emission and the temperature will evolve according to

$$
\frac{\stackrel{\mathrm{T}}{\mathrm{t}}}{\mathrm{r}}=-\frac{L}{\mathrm{U} / \mathrm{T}}=-\frac{g \mathcal{A} \sigma}{3 \mathcal{U}} \xi m^{-1} T^{2} .
$$

Therefore, the temperature is given by

$$
T_{\mathrm{c}}=T_{0}\left[1+\frac{g \mathcal{A} \sigma T_{0}}{3 \mathcal{U} \xi^{-1} m} t\right]^{-1}
$$

where $T_{0}$ and $t$ are the temperature at which, and time since, radiative cooling began. This gives a typical cooling timescale for gravastars of

$$
t_{\text {cool }}=\frac{3 \mathcal{U} \xi^{-1} m}{g \mathcal{A} \sigma T_{0}}=3.5 \times 10^{18} \frac{m}{\xi T_{0}} \mathrm{yr},
$$

which is generally quite long, unless $\xi$ is very large.

\section{Observational Constraints upon $\xi$}

The observational constraints we derive for $\xi$ arise from upper limits on the temperature, $T$, of black hole candidates, obtained through observations. That is, if the gravastar model is a proper description of the endpoint of gravitational collapse, it should result in a thermally emitting surface whose emission is below all limits set by spectral observations. Generally, each observed flux $F_{\nu \text { obs }}$ at frequency $\nu$ places a limit on $T$ via the condition

$$
F_{\nu \text { obs }}>F_{\nu}(T)=\frac{2 h \nu^{3}}{c^{2}} \frac{\mathrm{e}^{-h \nu / k T}}{1-\mathrm{e}^{-h \nu / k T}} \frac{\mathcal{A}}{D^{2}} m^{2},
$$

where $D$ is the distance to the compact mass.

As mentioned earlier, a putative gravastar would almost certainly have acquired most of its mass via accretion, either as part of its birth (e.g., during core collapse in a supernova explosion followed by the rapid accretion of a fallback disk) or over an extended period of time after birth (e.g., via accretion in a binary system or as an active galactic nucleus). Thus we expect $\Delta m / m \sim 1$.

If most of the mass was accumulated via rapid accretion, the temperature would have risen to the value given in equation (3). For any particular version of the gravastar 
Table 1. UV and X-ray Flux Limits on XTE J1118+480.

\begin{tabular}{cccc}
\hline$\nu(\mathrm{Hz})$ & $F_{\nu}\left(\mathrm{ergcm}^{-2} \mathrm{~s}^{-1}\right)$ & $T_{\max }(\mathrm{K})$ & Ref. \\
\hline $2.3 \times 10^{15}$ & $2.4 \times 10^{-30}$ & $4.3 \times 10^{5}$ & (McClintock et al. 2004) \\
$7.3 \times 10^{16}$ & $5.6 \times 10^{-32}$ & $2.7 \times 10^{5}$ & (McClintock et al. 2004) \\
$2.5 \times 10^{17}$ & $1.4 \times 10^{-32}$ & $6.7 \times 10^{5}$ & (McClintock et al. 2004) \\
\hline
\end{tabular}

model, if this $T_{\mathrm{h}}$ is less than the observational limit $T_{\text {limit }}$, then the model is obviously consistent with observations. Even if $T_{\mathrm{h}}$ exceeds $T_{\text {limit }}$, if the cooling time given in equation (8) is shorter than the age of the system, which we may take conservatively to be $t=15 \mathrm{Gyr}$ (the age of the universe), the model is again consistent since the gravastar would have had time to cool below $T_{\text {limit }}$. Therefore, as seen in Figure 1, the parameter space of the gravastar model is divided into three regions corresponding to when (i) accretion heating is insufficient to raise the temperature above detectable limits (labeled as "Insufficient accretion heating" in Figures 11 \& 2), (ii) accretion heating is sufficient to heat the surface above, and subsequent post-accretion cooling in $15 \mathrm{Gyr}$ is insufficient to reduce it below, detectable limits (labeled as "Excluded by Observations"), and (iii) when post-accretion cooling is sufficient to reduce the temperature below detectable limits in 15 Gyr (labeled as "Sufficiently rapid cooling"). Region (ii) is clearly ruled out by the observational constraint. We now apply this constraint via two black hole candidates with very stringent flux limits.

\subsection{XTE J1118+480}

The black hole candidate XTE J1118+480 is notable for its extraordinarily low accretion luminosity (McClintock et al. 2004), which allows us to place sensitive limits upon any X-ray flux from the vicinity of the horizon. At a distance of $1.8 \mathrm{kpc}$, the observed flux limits place an upper limit upon the temperature (as measured at infinity) of any horizon-sized surface of $T_{\text {limit }} \leq 2.7 \times 10^{5} \mathrm{~K}$ (see Table 1). With a mass of approximately $8 M_{\odot}$, this excludes the shaded region shown in Figure 1. In particular, we can exclude $\xi$ of order unity if as little as $4 \%$ of the mass of the object was accreted some time during its lifetime! However, due to the low mass, and correspondingly lower heat capacity (eq. 1), XTE J1118+480 does not constrain values of $\xi$ above $\sim 5 \times 10^{3}$.

\section{2. $\operatorname{Sgr} A^{*}$}

Being far more massive at $3.7 \times 10^{6} M_{\odot}$ (Schödel et al. 2003, Ghez et al. 2005a), the accretion-heated temperature of Sgr $A^{*}$ is necessarily lower than that for J1118+480. Nevertheless, the near-infrared (NIR) flux limits that have been placed upon Sgr A* are sufficiently stringent to limit the brightness temperature of any surface emission to less than $1.1 \times 10^{3} \mathrm{~K}$ (Table 2). As a consequence, values for $\xi$ between approximately $10^{4}$ and $10^{11}$ are excluded (see Figure 2) as long as Sgr $\mathrm{A}^{*}$ has accreted a reasonable fraction of its mass some time within the last $15 \mathrm{Gyr}$. 


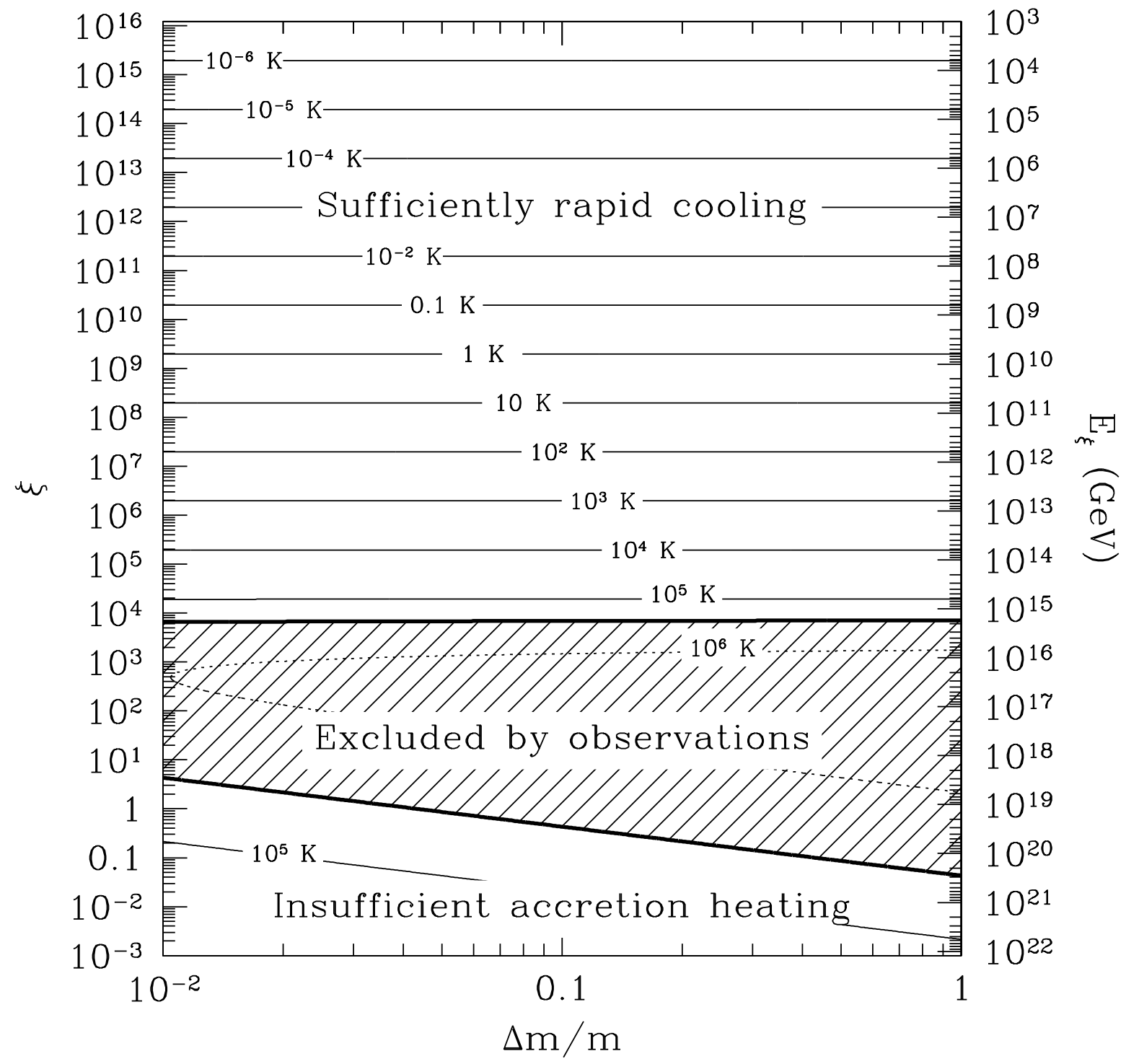

Figure 1. Contours of the predicted surface temperature today of a gravastar of mass $8 M_{\odot}$ heated by an episode of rapid accretion $15 \mathrm{Gyr}$ ago as a function of $\xi$ and the fractional mass accreted. The hatched region of the plot is excluded by observations of the black hole candidate XTE J1118+480.

\subsection{Sgr A*: Steady Accretion}

Additional constraints may be obtained from the fact that Sgr A* is presently accreting. If the observed radio/sub-mm luminosity of $10^{36} \mathrm{erg} \mathrm{s}^{-1}$ of the source is accretion powered with a canonical quasar radiative efficiency of $10 \%$, the present accretion rate of gas must be at least $2 \times 10^{-10} M_{\odot} \mathrm{yr}^{-1}$ (see, e.g., Broderick \& Narayan 2006). In fact, typical radiatively inefficient accretion flow models (RIAFs) imply accretion rates two orders of magnitude higher $\left(\sim 10^{-8} M_{\odot} \mathrm{yr}^{-1}\right.$, see, e.g., Narayan et al. 1995, Yuan 
Observational limits on gravastars

Table 2. Near-Infrared Flux Limits on Sgr A*.

\begin{tabular}{llll}
$\lambda(\mu \mathrm{m})$ & $F_{\nu}\left(\mathrm{ergcm}^{-2} \mathrm{~s}^{-1}\right)$ & $T_{\max }(\mathrm{K})$ & Ref. \\
\hline & & & \\
1.6 & 11 & $2.4 \times 10^{3}$ & (Stolovy et al. 2003) \\
2.1 & 2.8 & $1.6 \times 10^{3}$ & (Ghez et al. 2005b) \\
3.8 & 1.28 & $1.1 \times 10^{3}$ & (Ghez et al. 2005b) \\
4.8 & 3.5 & $1.7 \times 10^{3}$ & (Clénet et al. 2004) \\
\hline
\end{tabular}

et al. 2003). Finally, if stellar capture events are included the average accretion rate can be as high as $10^{-5}$ to $10^{-3} M_{\odot} \mathrm{yr}^{-1}$ (Magorrian \& Tremaine 1999).

Generally, the constraints that these accretion rates place upon $\xi$ depend on the time over which the rates have been maintained (which translates to a given value of $\Delta m / m)$. A natural estimate for this time scale (especially in the context of stellar captures) is the age of the Galaxy, roughly $10 \mathrm{Gyr}$, and thus implies $\Delta m / m$ of $5 \times 10^{-7}$, $3 \times 10^{-5}$ and $3 \times 10^{-1}$ for $\dot{M}$ of $2 \times 10^{-10} M_{\odot} \mathrm{yr}^{-1}, 10^{-8} M_{\odot} \mathrm{yr}^{-1}$ and $10^{-4} M_{\odot} \mathrm{yr}^{-1}$, respectively. Via equations (3) \& (9), this places lower limits upon $\xi$ of approximately $10^{9}, 3 \times 10^{7}$ and $2 \times 10^{3}$, for minimal, RIAF and stellar capture accretion rates, respectively, and are shown in Figure 3 ,

For continuous accretion the gravastar will not have had an opportunity to cool. In contrast, stellar capture events produce transient rapid accretion and allow the object to cool over the intercapture timescales $\left(\sim 10^{4} \mathrm{yr}\right)$. The upper limit implied by efficient cooling in this case corresponds to $\xi \lesssim 10^{18}$, which is already ruled out by the continuous gas accretion even at the minimal level.

\subsection{Total}

The combined limits imposed by XTE J1118+480 and Sgr A* are shown in Figure 4 . We see that all values of $\xi$ larger than unity are ruled out. In other words, presentday astronomical observations rule out modifications of general relativity of the kind described by Chapline et al. (2003) on all scales larger than the Planck length!

\section{Discussion}

Due to the long-range correlations inherent in gravastar theories, the heat-capacities of supermassive black holes will typically be significantly larger than expected for normal hadronic matter. As a consequence, steady-state arguments of the kind presented in Broderick \& Narayan (2006) may not be directly applicable. Nevertheless, we find that it is possible to strongly constrain the parameters of gravastar models with essentially similar considerations.

A generic feature of gravastar theories is the necessity of a spacetime phasetransition at large energies, required to facilitate the creation of the exterior, strongly 


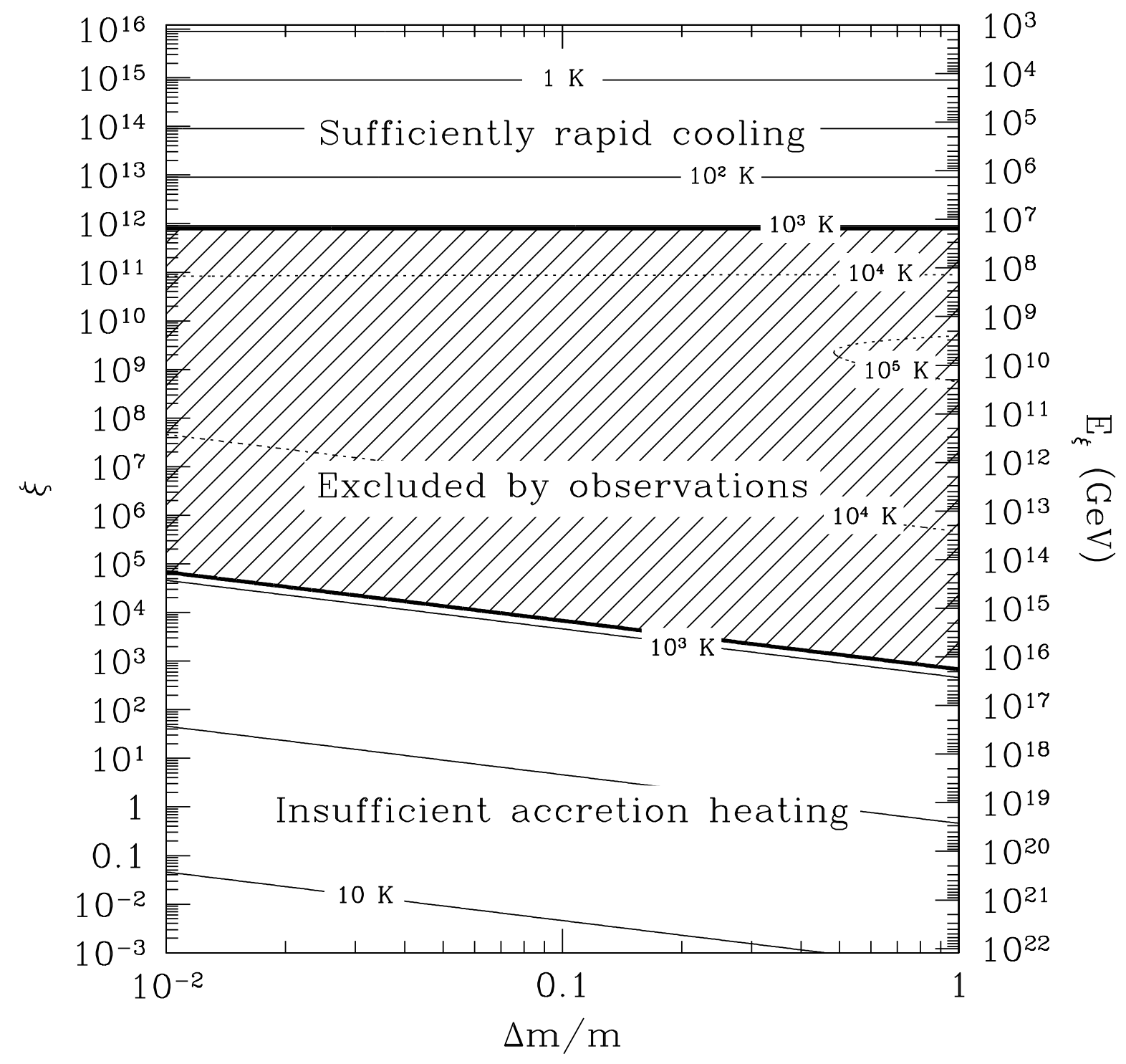

Figure 2. Contours of the predicted surface temperature today of a gravastar of mass $3.7 \times 10^{6} M_{\odot}$ heated by an episode of rapid accretion $15 \mathrm{Gyr}$ ago as a function of $\xi$ and the fractional mass accreted. The hatched region of the plot is excluded by observations of the black hole candidate Sgr A*.

correlated matter shell and interior anti-de Sitter space. Given that the physics associated with such a phase transition requires unknown modifications of general relativity, it is difficult to rule out all possible variants of the concept. However, in the context of the most physically motivated example to date, viz., the model of Chapline et al. (2003), it is possible to constrain quite strongly the parameters of the postulated phase transition. In particular, we find that the length scales over which modifications are allowed must be smaller than the Planck length at which, presumably, quantum gravitational effects enter in any case. 


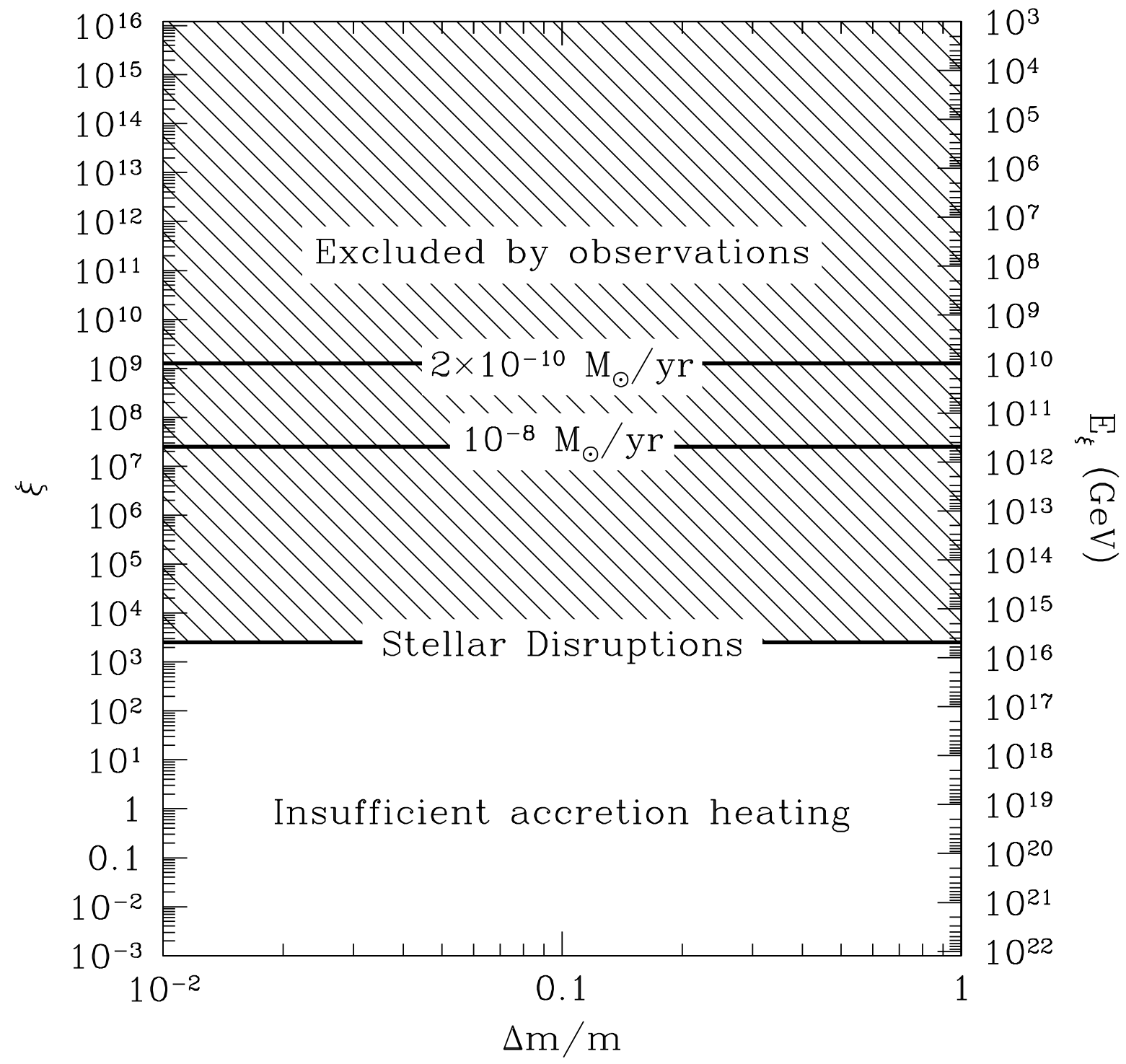

Figure 3. Excluded values of $\xi$ as a result of the presently observed steady accretion onto Sgr A*, which for the purposes of this plot is assumed to have continued for the past 10 Gyr.

More generally, the lack of discernible thermal emission from the surfaces of known black hole candidates provides a strong observational constraint on any alternate gravitational theory that does not admit horizons.

\section{Acknowledgments}

This work was supported in part by NASA grant NNG04GL38G. A.E.B. gratefully acknowledges the support of an ITC Fellowship from Harvard College Observatory. 


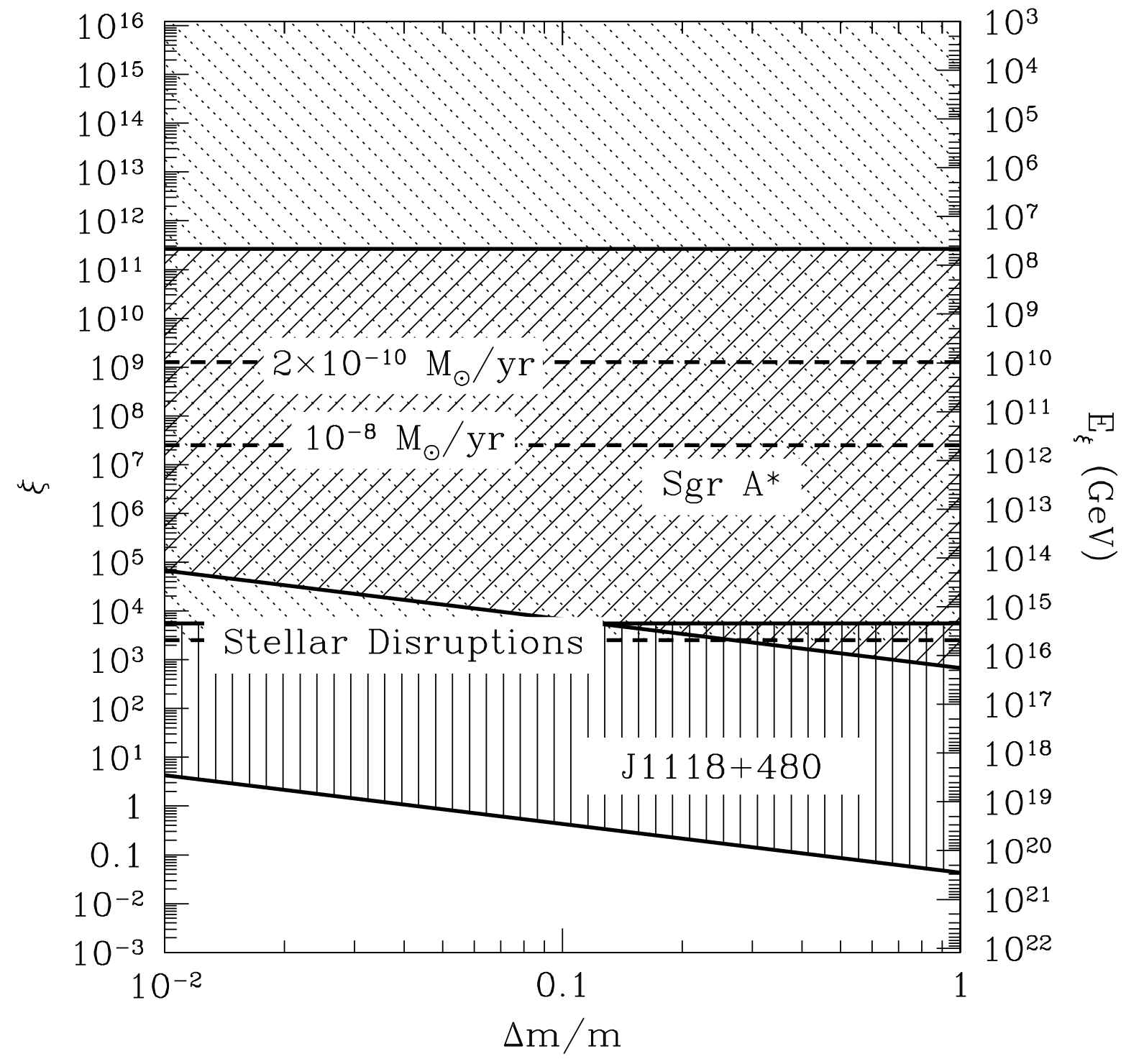

Figure 4. The currently excluded regions of the $\xi-\Delta m / m$ parameter space. The vertically hatched region is excluded by observations of XTE J1118+480 (Figure 1). The solid-diagonal hatched region is excluded by observations of Sgr A* (Figure 2). Finally, the the horizontal dashed lines show the lower limits placed upon $\xi$ by steady accretion onto Sgr A* (Figure 3), which for the purposes of this plot is assumed to have continued for the past $10 \mathrm{Gyr}$.

Broderick A E \& Narayan R 2006 ApJL 638, L21-L24.

Carter B M N 2005 Classical and Quantum Gravity 22, 4551-4562.

Chapline G et al. 2003 International Journal of Modern Physics A 18, 3587-3590.

Clénet $Y$ et al. 2004 A $B A$ 424, L21-L25.

Di Matteo T, Springel V \& Hernquist L 2005 Nature 433, 604-607.

Fryer C L, Heger A, Langer N \& Wellstein S 2002 ApJ 578, 335-347.

Ghez A M et al. 2005a ApJ 620, 744-757. 
Ghez A M et al. $2005 b$ astro-ph/0508664 .

Lobo F S N 2006 Classical and Quantum Gravity 23, 1525-1541.

Magorrian J \& Tremaine S 1999 MNRAS 309, 447-460.

Mazur P O \& Mottola E 2001 gr-qc/0109035 .

McClintock J E, Narayan R \& Rybicki G B 2004 ApJ 615, 402-415.

Narayan R 2005 New Journal of Physics 7, 199-+.

Narayan R, Yi I \& Mahadevan R 1995 Nature 374, 623-+.

Schödel R et al. 2003 ApJ 596, 1015-1034.

Soltan A 1982 MNRAS 200, 115-122.

Stolovy S, Melia F, McCarthy D \& Yusef-Zadeh F 2003 Astronomische Nachrichten Supplement 324, 419-423.

Visser M \& Wiltshire D L 2004 Classical and Quantum Gravity 21, 1135-1151.

Woosley S E, Heger A \& Weaver T A 2002 Reviews of Modern Physics 74, 1015-1071.

Yu Q \& Tremaine S 2002 MNRAS 335, 965-976.

Yuan F, Quataert E \& Narayan R 2003 ApJ 598, 301-312. 